\title{
Morphometric analysis of breast carcinoma: association with survival
}

\author{
D M PARHAM, A J ROBERTSON, R A BROWN* \\ From the Departments of Pathology and ${ }^{*}$ Mathematical Sciences, University of Dundee, Scotland
}

SUMMARY In a retrospective study of 126 cases of infiltrating ductal adenocarcinoma of the breast the percentage area of sections of each neoplasm occupied by malignant cells was assessed by morphometry. The duration of postoperative survival was related to the area occupied by tumour cells within a neoplasm; the greater the tumour cell area to stroma the more prolonged the survival. This variable seemed to be independent of histological grade and was of greater prognostic importance than the diameter of the neoplasm. Furthermore, combination of percentage of tumour area with histological grade provided greater information on the survival characteristics of these patients. Extensive tumour necrosis was a particularly poor prognostic feature.

In an attempt to predict postoperative survival of an individual patient with breast carcinoma pathologists have examined a variety of tumour features. The most widely accepted histological classification presently in use, introduced by Bloom and Richardson,' subjectively grades tumours by assessment of tubule differentiation, mitotic rate, and nuclear characteristics, although this subjective grading method has been shown to lack interobserver reproducibility. ${ }^{2}$ Others have since attempted to quantify the degree of nuclear pleomorphism and relate this to recurrence or survival $^{34}$ : their findings indicate a better prognosis with elongated ellipsoid, rather than rounder, nuclei. It has also been recognised that the outcome for the patient may depend on the host response to the tumour, ${ }^{5}$ but this aspect of tumour pathology has received little attention. Sistrunk and MacCarty originally assessed qualitatively the connective tissue and lymphocyte content within breast tumours. ${ }^{6}$ Others have since examined the local immunological reactions around tumours with regard to the cellular infiltration and changes within the regional lymph nodes. ${ }^{5-9}$ Maximum neoplasm diameter is also known to be important in determining the duration of survival. ${ }^{37910}$ Only one study has examined the actual percentage area of a neoplasm occupied by malignant cells, ${ }^{11}$ and it showed a considerable variation $(2 \cdot 9-$ $42 \cdot 8 \%$ ). It seemed reasonable, therefore, to examine whether the actual percentage of malignant cells within a neoplasm would prove a better guide to prognosis than tumour diameter alone.

Accepted for publication 5 August 1987

\section{Material and methods}

One hundred and twenty six women, mean age 67 years (range 41-96) were selected from the Cancer Registry. They had been consecutively diagnosed with primary infiltrating ductal adenocarcinoma of the breast and treated at Maryfield Hospital and Dundee Royal Infirmary in 1972 and 1973. Follow up for the purpose of this study stopped in April 1985. Information regarding the menstrual state of the patients was not available. Surgical treatment consisted of simple mastectomy and radiotherapy (104 cases), simple mastectomy (11 cases), wedge resection with radiotherapy (eight cases), or wedge resection alone (three cases). At the end of the follow up 66 patients had died and the survival time for each patient was recorded. For assessment of the actual percentage area of neoplasm occupied by tumour cells, 43 patients had to be excluded either due to recorded loss of original tissue from files (nine cases), insufficient remaining tissue for analysis ( 25 cases), or because of extensive tumour necrosis in the sections available (nine cases).

\section{MICROSCOPY}

The original haematoxylin and eosin stained sections were used for light microscopy and examined without knowledge of the patients' survival. The sections studied represented the largest area available incorporating both the periphery and centre of the tumour. The tumours were graded by two observers according to the method introduced by Bloom and Richardson ${ }^{1}$ and described in detail by Elston. ${ }^{12}$ The following aspects were graded on a three point scale: tubule 
formation; nuclear size; pleomorphism; and mitotic rate. A score of 3 to 5 formed grade 1 (well differentiated); 6 and 7 , grade 2 (moderately differentiated); 8 and 9 , grade 3 (poorly differentiated). Where variance between the observers on particular features occurred, a concensus decision was reached by review and discussion.

The proportion of malignant cells within a neoplasm was estimated microscopically by point counting using a graticule with 100 regularly distributed points at a total magnification of 100 . Due to the non-uniform distribution of malignant cells throughout a neoplasm consecutive non-overlapping fields were counted across the neoplasm from periphery to the centre and back. Depending on the size of the neoplasm, this was repeated until a total of 30 fields had been examined. The mean value was calculated and the results expressed as a percentage area of the neoplasm occupied by tumour cells. A preliminary study counting up to 60 consecutive non-overlapping fields in six different breast neoplasms was carried out: this indicated that an accurate result for percent of tumour area could be obtained by measurement of 30 consecutive fields (fig 1).

Statistical analysis of survival was carried out using life tables and the log rank test as outlined by Peto et $a l .{ }^{13}$ In each histological grade percentage of tumour area against survival was also assessed and a standard exponential distribution model applied. ${ }^{14}$

\section{Results}

No difference in the mean neoplasm size was noted between the 83 cases used to assess percentage of tumour area and the group excluded due to lost or

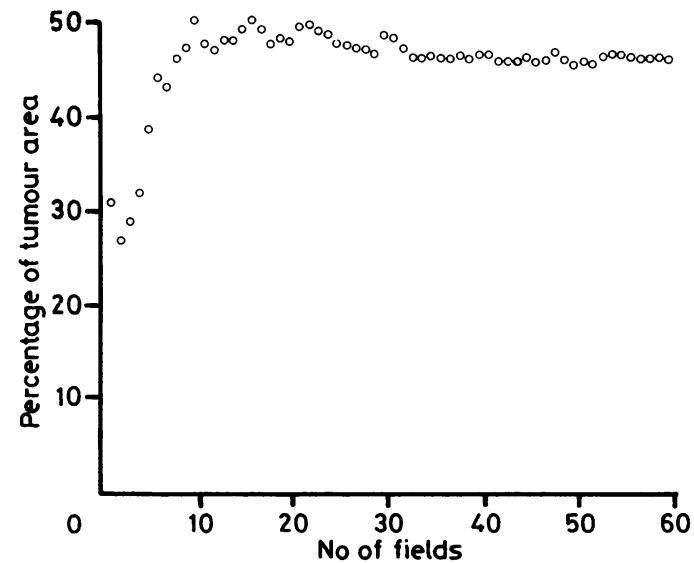

Fig 1 Cumulative average percentage of tumour area over 60 consecutive non-overlapping fields in typical breast carcinoma. insufficient material (mean tumour size $30 \mathrm{~mm}$ and 29 mm, respectively, Student's $t$ test: $\mathrm{t}=0.28, \mathrm{p}<0.8$ ). Similarly, a life table analysis (not shown) showed no significant difference in the survival pattern between the groups, indicating that no bias of the data would result from the excluded cases (log rank test: $\chi^{2}=0.185$ with $1 \mathrm{df}, \mathrm{p}>0.8$ ). By contrast, the life table in fig 2 shows a significant difference in the mortality pattern between the 83 cases used in this study to assess percentage of tumour area and those neoplasms which exhibited extensive necrosis (p $\leqslant 0.002$ ). It was found, however, that this latter group had on average slightly larger neoplasm diameters, although this difference was not significant

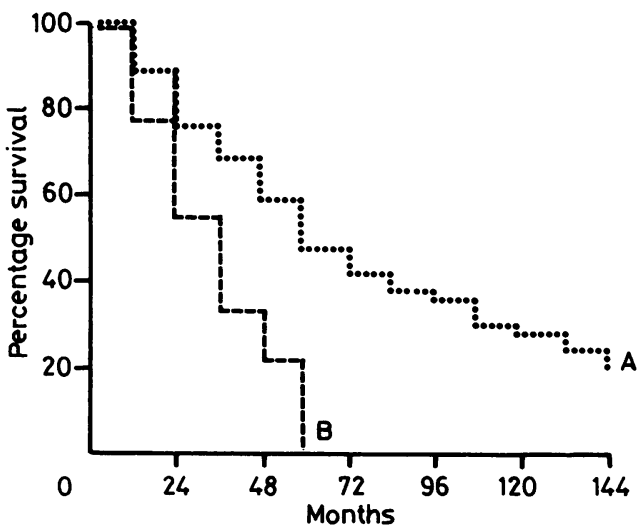

Fig 2 Life table showing mortality pattern between carcinomas exhibiting minimal necrosis $(A)$ and those with extensive necrosis $(B)$.

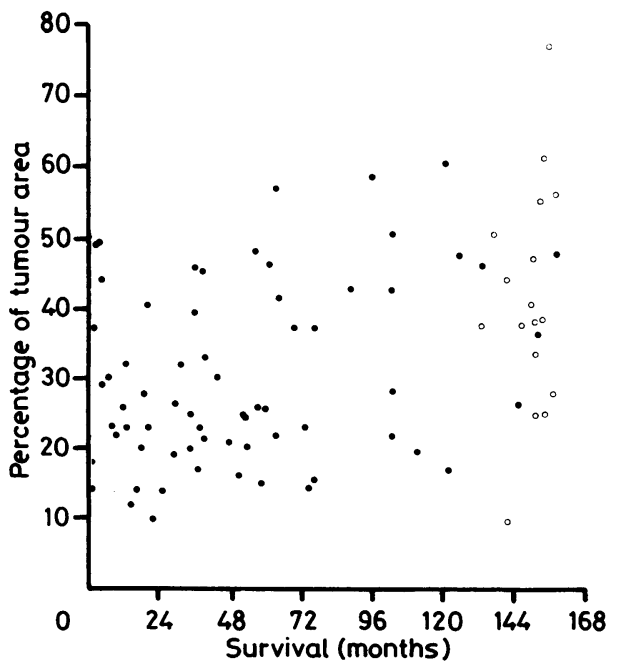

Fig 3 Scatter diagram showing association between percentage of tumour area and survival $(0=$ patients alive at the end of follow up). 
in the small number available for study (mean $=39$ mm, Student's $t$ test; $\mathrm{p}<0 \cdot 2$ ).

The percentage area of tumour in the 83 cases studied ranged from 9.5 to $77 \%$ (mean $=28.9 \%$; $\mathrm{SD}=14 \cdot 2 \%$ ). The scatter diagram (fig 3 ) showed a relatively low percentage of tumour area for patients with a short survival when compared with those with a long survival. Examination of the 25th, 50th, and 75th percentile of the percentage of tumour area showed that 21 patients had a tumour area less than and including $21.8 \%$ (group 1), 21 patients had a percentage of tumour area between 21.9 and $28.9 \%$ (group 2), 21 patients between 30 and $44 \cdot 2 \%$ (group 3), and 20 a percentage of tumour area greater than $44.4 \%$

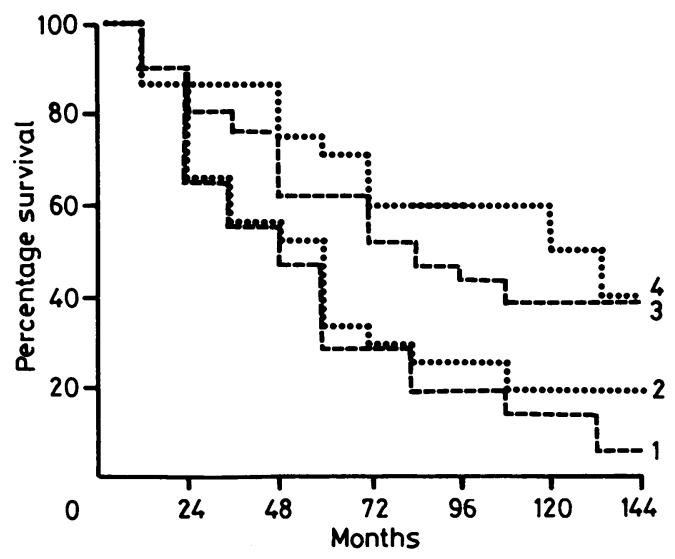

Fig 4 Survival curves at different percentage of tumour areas (group $1 \leqslant 21 \cdot 8 \%$, group $2 \leqslant 28.9 \%$, group 3 $\leqslant 44 \cdot 2 \%$, group $4>44 \cdot 4 \%$. Log rank test $\chi^{2}=9 \cdot 43 . d f=3$, $p<0.025$ ).

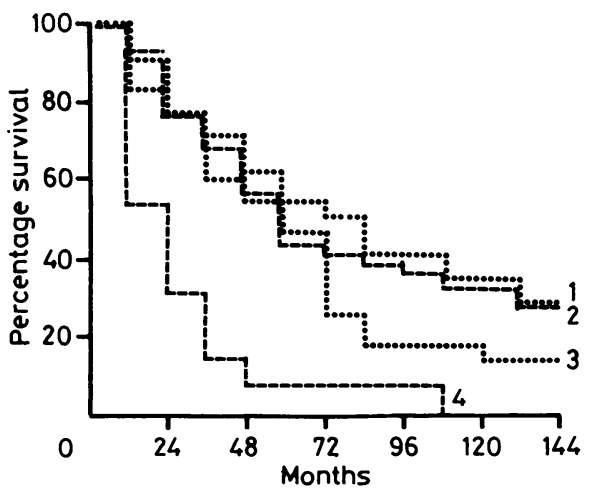

Fig 5 Survival curves showing mortality characteristics at different tumour diameters (group $1<20 \mathrm{~mm}$, group 2 $<40 \mathrm{~mm}$, group $3<60 \mathrm{~mm}$, group $4 \geqslant 60 \mathrm{~mm}$. Log rank test $\left.\chi^{2}=6 \cdot 853, d f=3, p<0 \cdot 1\right)$.
Table Composite survival index

\begin{tabular}{lll}
\hline & $\begin{array}{l}\text { Probability of } \\
\text { survival } \geqslant 48 \\
\text { months }\end{array}$ \\
\cline { 2 - 3 } Exponential distribution model & $10 \%$ & $50 \%$ \\
\hline Grade $1 \log \left(\mathrm{S}_{1}(\mathrm{t}\right.$, area $\left.)\right)=\mathrm{te}^{-3.66-0.04}$ area & 0.44 & 0.85 \\
Grade $2 \log \left(\mathrm{S}_{1}(\mathrm{t}\right.$, area $\left.)\right)=\mathrm{te}^{-3.51-0.02}$ area & 0.31 & 0.59 \\
Grade $3 \log \left(\mathrm{S}_{1}(\mathrm{t}\right.$, area $\left.)\right) \times \mathrm{te}^{-2.38-0.055}$ area & 0.098 & 0.84 \\
\hline
\end{tabular}

Grade 1, SE of established variables: $-3.66(0.64)-0.04(0.02)$

Grade 2, SE of established variables: $-3.51(0.36)-0.02(0.01)$

Grade 3, SE of established variables: $-2 \cdot 38(0.64)-0.065(0.02))$

$\mathrm{S}(\mathrm{t})=$ probability that individual survives at least $\mathrm{t}$ months.

(group 4). Fig 4 shows the cumulative proportion surviving (the proportion alive at the beginning of each year) with significantly different mortality patterns between each of these groups $(p<0.025)$. Greater significance between mortality and percentage of tumour area $(0.01>p>0.001)$ can be achieved by dividing percentage of tumour area into two larger groups (group $1 \leqslant 28.9 \%$, group $2 \geqslant 30 \%$ ).

The percentage of tumour area showed no positive correlation with either tumour differentiation or maximum tumour diameter. It was noted, however, that as the maximum tumour diameter increased the prognosis became worse, although the results from the life table in fig 5 do not quite achieve significance when tumour size $(<20,<40,<60, \geqslant 60 \mathrm{~mm})$ is matched against survival $(\mathrm{p}<0 \cdot 1)$.

Histological grade significantly correlated with survival ( $\mathrm{p}<0.005$, results not shown). An exponential curve fits the distribution of survival times in histological grade 1 , and similar curves can be fitted to the other histological grades (table). The analysis was performed using the GLIM statistical package ${ }^{15}$ to implement the methods described by Aitken and Clayton. ${ }^{14}$ The goodness of fit of the exponential distribution was assessed by comparing the deviance produced by the GLIM package with the relevant percentage points of the $\chi^{2}$ distribution. The observed survival distributions for histological grades 1 and 3 were found to be not significantly different from the exponential distributions in both cases (grade 1, deviance $=34 \cdot 6, \mathrm{df}=32, \mathrm{p}>0 \cdot 1$; grade 3 , devian$c e=15 \cdot 7, \mathrm{df}=10, \mathrm{p}>0 \cdot 1)$. For grade 2 there was some evidence of a genuine discrepancy between the observed survival distribution and the fitted exponential distribution (deviance $=63.7, \mathrm{df}=35, \mathrm{p}<0.01$ ). The probability of surviving a certain period can be derived from these curves within each histological grade (table). For a grade 1 patient with a $50 \%$ tumour area, the estimated probability of surviving 48 months is 0.85 , roughly twice the survival probability for a patient with a $10 \%$ tumour area neoplasm $(0 \cdot 44)$. In grade 3 the difference is even more striking. 


\section{Discussion}

The results indicate a very wide range in the proportion of tumour to stroma between neoplasms, supporting the original observations of Underwood. " In particular, the percentage of tumour area may be of predictive value with regard to long term prognosis; neoplasms with a high proportion of tumour to stroma having a better prognosis than those neoplasms with a low proportion of tumour to stroma. Death occurred most rapidly in patients with a low percentage of tumour area over the first seven years. The product of percentage of tumour area and maximum tumour diameter did not form a more accurate prognostic index.

The percentage of tumour area, it should be noted, does not necessarily reflect the degree of tumour cellularity, as a variation in cell size between neoplasms could account partly for the results seen. Nevertheless, it would be reasonable to conclude that the percentage of tumour area represents a number of inter-related or independent aspects of tumour growth; a high proportion of malignant cells within a neoplasm may reflect a greater degree of tumour to tumour cell adherence and a reduced ability to metastasise, but many single cells within a neoplasm could give the same percentage tumour area as several large cellular masses.

A further potential factor contributing to the percentage of tumour area is the amount of stroma produced within the neoplasm. Stromal production may be indicative of the host defense produced in response to the degree of tumour dedifferentiation and hence malignancy. Furthermore, the amount of fibrous tissue produced may indicate the age of the neoplasm with regard to the natural history of the disease. No association was found between the percentage tumour area and histological grade, which is presently believed to reflect tumour differentiation or the maximum diameter of the tumour mass. There is, however, experimental evidence produced by Gullino and Grantham ${ }^{16}$ to suggest that the neoplastic cells themselves regulate the connective tissue content in a neoplasm, and recently it has been shown that murine mammary carcinoma may induce oncogene expression with an associated increase in growth rate in cultured fibroblasts. ${ }^{17}$

Previous assessment of the cellular and stromal components of tumours has produced conflicting views. Baak et al noted a positive correlation between a high cellularity and survival, when assessing at the tumour periphery over five fields (at $\times 1000),{ }^{3}$ but they failed to discuss the clinical importance. Hamlin ${ }^{5}$ and Alderson $e t a l$ also noted that patients with tumours containing dense collagenous stroma, and presumably having a low cellularity, had a worse outcome. In contrast, Sistrunk and $\mathrm{MacCarty}^{6}$ in their study regarded stromal hyalinisation as a favourable feature, and Black et $a l^{18}$ found that by semiquan $-\because$ titative assessment a greater cellularity correlated with a poorer survival. Nevertheless, Dawson et al, ${ }^{12} \frac{}{6}$ reviewing the histological results of patients witho breast cancer surviving 25 years with a control group $\overline{\bar{s}}$ dying in less than 10 years, found no difference in the degree of fibrosis when assessed subjectively.

This paper makes no attempt to differentiate between the different stromal components, which, on a. quantitative basis, may provide further information. $\overrightarrow{\vec{\omega}}$ Okamoto performed a histochemical study of breast ${ }^{\omega}$ carcinoma and other tumours and concluded thatot? mature thick collagenous stroma represented a defensive reaction, while growing immature stroma adjacent to invading tumour may have a promotive or $\rightarrow$ defensive role. ${ }^{20}$ More recently the presence of elastosis $\omega$ in breast tumours has been associated with a favour-o able prognosis, ${ }^{21}$ although others have been unable to confirm this finding. ${ }^{22}$

The wide variation in the proportion of tumour cellso within a mass also casts doubts on the clinical importance of breast tumour oestrogen receptor status, presently performed on tissue homogenates $-\vec{\bullet}$ high receptor values being associated with a better ${ }_{\infty}^{\infty}$ prognosis. ${ }^{23}$ A positive correlation between oestrogen receptor values and cellularity has previously beeno shown. ${ }^{24}$ The oestrogen receptor content of a neo-plasm may therefore indirectly partly reflect tumouro cellularity and hence prognosis. Black et al still found $\frac{\mathbb{}}{\odot}$ that receptor positive tumours had a better prognosis $\varrho$ following statistical correction of tumour cellularity, $\overrightarrow{\overrightarrow{0}}$ when assessed subjectively. ${ }^{18}$

Our results indicate little association between the percentage of tumour area and histological grade. This may partly be due to the wide variability in tumouro density which would considerably change the number: of neoplastic cells examined per high power field for 3 . the mitotic rate. Nevertheless, it is generally accepted that histological grade is only moderately predictive 3 for grades 1 and 3 tumours, with the survival patterno of grade 2 tumours being less certain. ${ }^{25}$ It is noteworthy that the combination of percentage of tumour areao and histological grade, particularly in grade 1 and 3 tumours, provides greater information on survivalos patterns.

Size of primary tumour has been shown to relate toN prognosis, ${ }^{37910}$ and our results, although not sig- $\omega$ nificant, would tend to support this. Others ${ }^{526} 27$ have failed to show any clear association. An unexpected result from this study was the strong correlationo? between the presence of extensive tumour necrosis and survival with no patient living more than five years. To

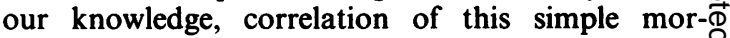
phological variable with survival has not previously 
been reported and may deserve further consideration. Necrosis, as previously stated by Fisher, ${ }^{28}$ tends to occur in the most malignant tumours, although in our small sample necrosis was evenly distributed among the histological grades (results not shown). Presumably these neoplasms rapidly outgrow their blood supply; this would explain the lack of difference in diameter between the necrotic and non-necrotic tumours as it is the rate of growth rather than overall size that is more important.

In conclusion, this paper has shown that the ratio of tumour cell area to stroma in breast cancer is important in relation to survival, the correlation of which is better than that found with maximum tumour diameter and survival. The combination of percentage of tumour area and histological grade also provided more detailed information on the survival characteristics of breast cancer patients. It follows that measurement of the ratio of tumour cell area to stroma ought to be considered when forming a prognostic index, especially as automated methods of quantitation are becoming readily available. Further refinement of the analysis may also be possible as some breast carcinomata are considered to be more cellular at the periphery than at the centre; the peripheral fields therefore form a greater proportion of the whole neoplasm. The morphometrical analysis performed by Underwood" took this into account, based on the assumption that the shape of a neoplasm approached that of a sphere. It is now considered that breast carcinomas tend to take the form of an ellipsoid. ${ }^{29}$ These factors ought to be considered in any prospective study when measurement of the dimensions of a neoplasm and histological sampling can be performed accurately.

We thank Professor J Swanson Beck for his support and $\mathrm{S} \mathrm{McPherson}$ for technical help.

\section{References}

1 Bloom HJG, Richardson WW. Histological grading and prognosis in breast cancer. Br J Cancer 1957;11:359-77.

2 Delides GS, Garas G, Georgouli G, et al. Intralaboratory variations in grading of breast carcinoma. Arch Pathol Lab Med 1982;106:126-8.

3 Baak JPA, Kurver PHJ, De Snoo-Niewlaat AJE, De Graeff S, Makkink B, Boon ME. Prognostic indicators in breast cancermorphometric methods. Histopathology 1982;6:327-39.

4 Van der Linden HC, Baak JPA, Lindeman J, Hermans J, Meyer CJLM. Morphometry and breast cancer 11. Characterisation of breast cancer cells with high malignant potential in patients with spread to lymph nodes: preliminary results. J Clin Pathol 1986;39:603-9.

5 Hamlin IME. Possible host resistance in carcinoma of breast: a histological study. Br J Cancer 1968;22:383-401.

6 Sistrunk WE, MacCarty WC. Life expectancy following radical amputation for carinoma of the breast-a clinical and pathological study of 218 cases. Ann Surg 1922;75:61-9.

7 Alderson MR, Hamlin I, Staunton MD. The relative significance of prognostic factors in breast carcinoma. Br J Cancer 1971; 4:646-56.
8 Blamey RW, Davies CJ, Elston CW, Johnson J, Haybittle JL, Maynard PV. Prognostic factors in breast cancer-the formation of a prognostic index. Clin Oncol 1979;5:227-36.

9 Haybittle JL, Blamey RW, Elston CW, et al. A prognostic index in primary breast cancer. Br J Cancer 1982;45:361-6.

10 Say CC, Donegan WL. Invasive carcinoma of the breast: prognostic significance of tumour size and involved axillary lymph nodes. Cancer 1974;34:468-71.

11 Underwood JCE. A morphometric analysis of human breast carcinoma. Br J Cancer 1972;26:234-7.

12 Elston CW. Assessment of histological grade in human breast cancer. In: Blamey RW, ed. Seminar in breast cancer. London: Update Publications, 1984:47-50.

13 Peto R, Pike MC, Armitage P, et al. Design and analysis of randomised clinical trials requiring prolonged observation of each patient. II Analysis and examples. Br J Cancer 1977;35: 1-39.

14 Aitkin M, Clayton D. The fitting of exponential, Weibull and extreme value distributions to complex censored survival data using GLIM. Appl Statist 1980;29:156-63.

15 Baker RJ, Nelder JA. General linear interactive modelling: Release 3. London: Royal Statistical Society, 1978.

16 Gullino PM, Grantham FH. The influence of the host and the neoplastic cell population on the collagen content of a tumour mass. Cancer Res 1963;23:648-53.

17 Horak E, McGee J. Coordination of tumour and stromal cell growth: Interaction with malignant cells results in oncogene expression in host stromal fibroblasts. J Pathol 1987;151:39A.

18 Black R, Prescott R, Bers K, Hawkins A, Stewart H, Forrest P. Tumour cellularity, oestrogen receptors and prognosis in breast cancer. Clin Oncol 1983;9:311-8.

19 Dawson PJ, Ferguson DJ, Karrison T. The pathologic findings of breast cancer in patients surviving 25 years after radical mastectomy. Cancer 1982;50:2131-8.

20 Okamoto $Y$. Stromal response in relation to invading forms of tumours: A histochemical and histopathological study. Gann 1966;57:563-76.

21 Shivas AA, Douglas JG. The prognostic significance of elastosis in breast carcinoma. $J$ R Coll Surg 1972;17:315-20.

22 Robertson AJ, Brown RA, Cree IA, MacGillivray JB, Slidders W, Beck JS. Prognostic value of measurement of elastosis in breast carcinoma. J Clin Pathol 1981;34:738-43.

23 Walt AJ, Singhakowinta A, Brooks SC, Cortez A. The surgical implications of estrophile protein estimations in carcinoma of the breast. Surgery 1976;80:506-12.

24 Underwood JCE, Dangerfield VJM, Parsons MA. Oestrogen receptor assay of cryostat sections of human breast carcinoma with simultaneous quantitative histology. J Clin Pathol 1983; 36:399-405.

25 Rich MA, Brennan MJ. The breast cancer prognostic program: A study of the metastatic process, In: Brennan MJ, McGrath CM Rich MA, Eds. Breast cancer: new concepts in etiology and control. New York: Academic Press, 1980:29-51.

26 Kunath CA. Problem of cancer of the breast-Radical mastectomy in 90 cases. Archives of Surgery 1949;41:66-78.

27 Hoopes BF, McGraw AB. The Halsted radical mastectomy-five year results in 246 consecutive operations at the same clinic. Surgery 1942;12:892-905.

28 Fisher ER, Gregorio RM, Fisher B, et al. The pathology of invasive breast cancer. A syllabus derived from the findings of the National Surgical Adjuvant Breast Project (protocol No 4). Cancer 1975;36:1-84.

29 Atkinson EN, Brown BW, Montague ED. Tumour volume, nodal status and metastasis in breast cancer women. $J N C I$ 1986 76:171-8

Requests for reprints to: Dr D M Parham, Department of Pathology, Ninewells Hospital \& Medical School, Dundee, Scotland DD1 9SY. 